\title{
Hepatic and Epicardial Fat as Early Strong Predictors for Metabolic Syndrome Mohammed Abdel-Hassib ${ }^{1}$, Hossam Elashmawy ${ }^{2}$ and Yasser Elsayed Mohammed ${ }^{3}$ \\ ${ }^{1}$ Internal Medicine Department, ${ }^{2}$ Clinical Pathology Department and ${ }^{3}$ Cardiology Department, Faculty of Medicine, Al-Azhar University \\ *Corresponding Author: Yasser Elsayed Mohammed, email: Yasserelsayed97_@yahoo.commobile:0020100122
}

\begin{abstract}
Introduction: hepatic steatosis (HS) has been previously considered as a valuable and long-established indicator for evaluation of metabolic syndrome (MetS) and cardio-metabolic risks. Recently, epicardial adipose tissue (EAT) has been proposed and up to be established to carry the same or more than of HS as regarding cardio- metabolic risks. Hence, both of HS and cardiac steatosis coexist and interact with each other i.e. one reflects to theother. There are little data assessing the associations of hepatic and epicardial fat in non-metabolic-syndrome Egyptian adults. Objective: to evaluate the relationship between HS and EAT, and its relation to the components of metabolic syndrome.

Patients and methods: We studied fifty HS patients accidentally discovered by abdominal ultrasound and fifty healthy matched controls with normal fat content in the liver (without HS) by ultrasound for comparison. Both groups underwent complete history taking, general examination, laboratory investigations and 2D transthoracic echo Doppler study at baseline and 18 months thereafter only for patient's group.
\end{abstract}

Results: this study showed that, there is a positive correlation between HS and EAT and metabolic syndrome. HS and EAT are early and sensitive predictors for metabolic syndrome.

Conclusion: hepatic and epicardial fat are early and strong predictors of metabolic syndrome.

Keywords: hepatic and epicardial fat, metabolic syndrome.

\section{INTRODUCTION}

Recently, there is growing awareness thatthe behavior of the accumulated fat is completely different from body compartment to another e.g. excessive adipose tissue accumulation in the subcutaneous, intramuscular, and retroperitoneal is considered a less metabolically harmful; i.e. less of insulin resistance, lipolysis rate, and steroidhormone receptors, than those organ where specific (Ectopic) fat accumulates and surrounds internal organs "visceral adiposity" [Central or truncal, hepatic, epicardial, and dorsocervical regions] ${ }^{[1]}$.

Obesity leads not only to increased fat depots in classical adipose tissue locations, but also to significant lipid infiltration within internal organs "visceral adiposity" [2].

Different visceral fat compartments have several systemic effects and may play a role in the development of both insulin resistance and cardiovascular diseases. In the last couple of years special attention has been paid to the epicardial adipose tissue (EAT), which can be quantified by non-invasive cardiac imaging techniques ${ }^{[3]}$.

Non alcoholic fatty liver disease (NAFLD) is caused by the excessive accumulation of fat in the liver and commonly associated with obesity and metabolic syndrome. NAFLD can be considered the hepatic expression of the metabolic syndrome ${ }^{[4]}$.
While hepatic steatosis (HS) was considered as a traditional indicator of metabolic syndrome (MetS), epicardial adipose tissue was proposed to carry high cardiometabolic risk compared to general fat accumulation ${ }^{[5]}$. Hence; both of HS and cardiac steatosis coexist and interact with each other (One reflects to the other i.e. epicardial fat reflects intraabdominal visceral fat). Nowadays and in the coming, both of HS and EAT becomes novel therapeutic target for MetS and cardiovascular risks ${ }^{[6]}$, such as high BP, coronary artery disease (CAD), dyslipidemia, carotid atherosclerosis, polycystic ovary syndrome (PCO), insulin resistance, and even type I DM associated with central obesity ${ }^{[7]}$.

However, lipid accumulation in cardiac myocytes is also an early sign of pathogenesis of diabetic cardiomyopathy ${ }^{[8]}$. Epicardial adipose tissue is a "novel" visceral fat depot with peculiar anatomic and biochemical features ${ }^{[2]}$.

Anatomically; EAT is a unique fat compartment, depot between the visceral pericardium and myocardium, sharing a common embryologic origin (brown adipose tissue and splancho-pleuric mesoderm during embryogenesis) and strong genetic dependence with the visceral fat $\operatorname{depot}^{[9]}$.

EAT and the myocardium does not haveany fascia separates, thereby sharing the same microcirculation (branches of coronary arteries). By 
the adult age, the distribution of EAT is asymmetric, as it settles at peri-vascular, inter-ventricular, and atrioventricular grooves extending to the apex ${ }^{[10]}$.

Functionally, EAT has been recently recognized as a highly active organ having a unique source of huge number of protective and harmful systemic effectors bioactive mediators e.g. proatherogenic and pro-inflammatory adipocytokines and chemokines e.g. adiponectin, transforming growth factor $\beta 1$, e-selectin, endothelin, and fibroblast growth factor, as well as free fatty acids (FFA), that may influence through endocrine, paracrine and vasocrine effects ${ }^{[11]}$.

Peculiar adipokine (vaspin) and FFA are linked to sympathetic over activity that influences the autonomic nervous system of the heart and impairs hyperemic stimulation of coronary artery flow reserve in patients with NAFLD. EAT is the greatest stores and release for FFA ${ }^{[8]}$.

It is well known that, high-sensitivity Creactive protein (hs-CRP) is a risk marker for coronary artery disease, and Ouchi and his colleagues described a strong negative association between adiponectin and hs-CRP, both in plasmaand in the adipose tissue of patients with atherosclerotic coronary disease ${ }^{[12]}$.

It has been proposed that; the EAT could act as a physiological buffer that protects the heart against cardio-lipotoxicity generated by FFA. Liang et al., reported that, differences in right atrioventricular groove EAT thickness can help differentiate the inflammatory status of obese nondiabetic men with MetS ${ }^{[13]}$.

Both cardiac and hepatic steatosis are measured (Quantified) by ultrasound; a simple and readily available, valid, rapid, inexpensive, most convenient, non-invasive and real method used in clinical practice for assay of ectopic fat accumulation. Computed tomography (CT), or particularly magnetic resonance imaging (MRI) was considered the gold standard way to detect and quantify epicardial fat. However, it is difficult to measure adipose tissue using CT or MRI because of cost-effectiveness, the application of radiation, and the use of contrast media. In addition, the using echois advantageous because other cardiac parameters can be achieved simultaneously ${ }^{[14]}$.

\section{SUBJECTS AND METHODS Subjects:}

- Fifty (HS) patients (25 men aged between 28-57 years and 25 women aged between $26-52$ years) with mean age $42.30 \pm 7.28$ years, accidentally discovered by abdominal ultrasound, were enrolled in the study.

- Fifty healthy matched controls (25 men aged between 28-51 years and 25 women aged between 27-55 years) with mean age $40.96 \pm 6.83$ years, with normal fat content in the liver by ultrasound forcomparison.

- All patients enrolled in the study were from outpatient clinic of Bab-Elsharyia University Hospital, Cairo, Egypt.

- The study started from the beginning of February 2017 to the end of September 2018.

\section{Exclusion criteria:}

- One or more of the clinical or investigational criteria of metabolic syndrome and International Diabetes Federation (IDF) risk factors for type 2DM:

- Central (visceral) obesity; Waist circumference $(\mathrm{WC}) \geq 102 \mathrm{~cm}$ in men and $88 \mathrm{~cm}$ in women.

- $\mathrm{BMI} ;>25 \mathrm{Kg} / \mathrm{m} 2$

○ Blood pressure $(\mathrm{BP}) \geq 140 / 85 \mathrm{mmHg}$ or on antihypertensive treatment.

$\circ$ Prediabetes; Fasting blood sugar (FBS) 100 -125 $\mathrm{mg} / \mathrm{dl}, 2$ hours postprandial blood sugar (2h-PPBS) 140-200 mg/dl and/or Hb A1c 5.7- 6.4\%.

○ Serum lipid abnormalities; hypertriglyceridemia, triglycerides $(\mathrm{TG}) \geq 150 \mathrm{mg} / \mathrm{dl}$, and/or HDLc $<50$ $\mathrm{mg} / \mathrm{dl}$.

- Acanthosis nigricans.

- Habitual physical inactivity and sedentary life.

- Polycystic ovary (PCO); Diagnosed through one or more of; concurrent history, clinical "oligomenorrhea, amenorrhea and/or ovulatory dysfunction", ultrasonography and/or biochemical hyperandrogenism.

- Hyperuricemia; serum uric acid $\geq 6 \mathrm{mg} / \mathrm{dl}$ in men and $\geq 5 \mathrm{mg} / \mathrm{dl}$ in women.

- Drug that would be able to affect metabolic variables (e.g. glucocorticoids, anticonvulsants, hypolipidemic agents and oral contraceptives), smoking, or modest to severe alcoholic history.

- Current or past history of evidenced endocrinopathies, chronic cardiovascular, respiratory, or hepatic diseases other than steatosis (with negative $\mathrm{HCV}-\mathrm{Ab}$ and $\mathrm{HBsAg}$ ).

- Exclusion criteria for controls were the same as that for patients.

Methods:

All participants were subjected to the following:

- Approval of the ethical committee of AlAzhar Faculty of Medicine was obtained.

- Written informed consent before enrollment. 
- A detailed history and physical examination: A brief medical history was taken to gather demographicdata, such as age, sex, and associated personal and family background. Weight and height were measured with the participants wearing only their underwear. BMI was calculated as weight in kilograms divided by height in meters squared. Using the IDF criteria, WC (in centimeters) was measured at its narrowest part of the body midway between the underside of the lowest rib and the iliac crests with subjects standing.

- BP was taken after at least 10 minutes of rest, using the auscultation method through a standard mercury sphygmomanometer. Three measurements were performed, and the average of the BP measurements was calculated.

- Laboratory investigations including: FBS, 2hPPBS, HbA1c, lipids profiles after a 12-hr overnight fast (T.Ch., TG, LDLc, and HDLc), albumin/creatinine ratio, serum uric acid, liver enzymes, Highsensitivity C-reactive protein (hs-CRP).

- Abdominal ultrasound (U/S): All participants underwent abdominal U/S to assess HS using an Acuson Sequoia (Siemens, Mountain View, CA) with a convex probe $(2.5-5 \mathrm{MHz})$. HS was assessed by qualitative visual scoring on a scale of $0-3(0=$ absent, $1=$ mild, $2=$ moderate, and $3=$ severe) on the basis of an [Abnormally intense, hyperechoic echogenicity of the hepatic parenchyma compared with the kidneys, blurring of blood vessel structures, and deep attenuation of the ultrasonic beam].

- Echocardiographic measurement of epicardial fat thickness (EFT): Standard two-dimensional echocardiography was performed in all participants using a $2.5-3.5 \mathrm{MHz}$ transducer.

Interventricular septal wall thickness and posterior wall thickness, dimension of the left ventricle (LV) cavity, and the LV mass index were assessed according to the criteria of the American Society of Echocardiography.

- Definition of EFT was considered as the echofree space (hypoechoic space) between the visceral layer of the pericardium and the outer wall of the myocardium anterior (vertical) to the right ventricular free at end-systole in three cardiac cycles' image in the left lateral decubitus position according to previous methods.

- Mean EFT overlying the right ventricle was calculated as average from the parasternal long axis and short axis views, which allows for the most accurate measurement. Because EFT measurement might be inconsistent with measurement locations. Two settings of echocardiographic at different time (Not more than one week) in some of cases.

- If measurements by the two-setting differed by $>5 \%$ for any of the variables, the patient was not included; if the difference was $<5 \%$, the measurements were averaged.

- The pervious laboratory and radiological parameters assayed at the base line for all participants and 18 months thereafter only for the cases.

- All participants in the case group were followed regularly throughout the study. A number of cases discontinued the follow up and therefore excluded with a final 50 cases completed the study.

\section{Statistical analysis}

The following statistical tests and parameters were used: mean \pm standard deviation (SD), the student's t-test to compare means, and the chi-squared test to compare frequencies.

\section{RESULTS}

Table (1): Demographic data of patients' and control groups.

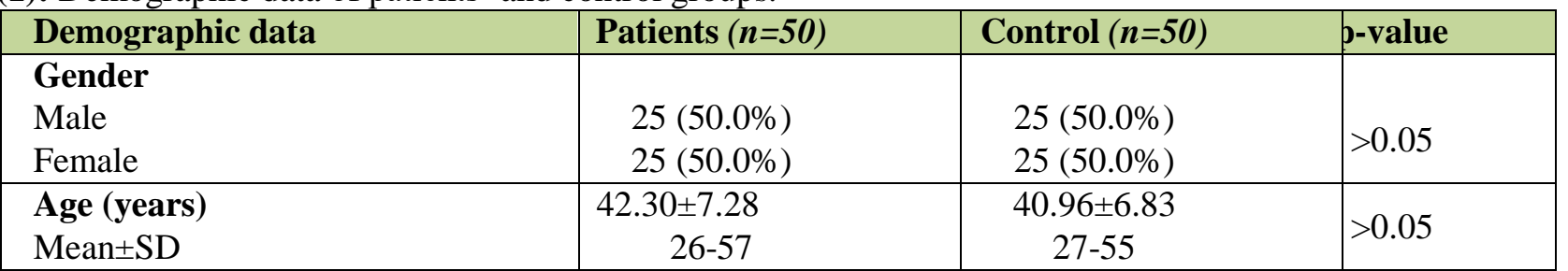

p-value $>0.05 \mathrm{NS}$

This table shows no statistically significant difference between groups according to demographic data. 
Table (2): Comparison of U/S data between baseline and after 18 months in patient's group.

\begin{tabular}{|l|c|c|c|}
\hline U/S degree of HS & At baseline & After 18 months & p-value \\
\hline I (Mild) & $10(20.0 \%)$ & $5(10.0 \%)$ & \multirow{2}{*}{$<0.05^{*}$} \\
\hline II (Moderate) & $30(60.0 \%)$ & $18(36.0 \%)$ & \\
\hline III (Severe) & $10(20.0 \%)$ & $27(54.0 \%)$ & \\
\hline
\end{tabular}

*p-value $<0.05 \mathrm{~S}$

This table shows statistically significant difference between baseline and after 18 months according to U/S.

Table (3): Comparison between patients' and control groups regarding the EFT at baseline.

\begin{tabular}{|l|c|c|c|}
\hline EFT at baseline & Patients $(\boldsymbol{n}=50)$ & Control $(\boldsymbol{n}=50)$ & p-value \\
\hline Normal $(<5 \mathrm{~mm})$ & $11(22.0 \%)$ & $50(100.0 \%)$ & \multirow{3}{*}{$0.001 * *$} \\
\hline Moderate $(5-7 \mathrm{~mm})$ & $33(66.0 \%)$ & $0(0.0 \%)$ & \\
\hline Marked $(>7 \mathrm{~mm})$ & $6(12.0 \%)$ & $0(0.0 \%)$ & \\
\hline
\end{tabular}

**p-value $<0.001 \mathrm{HS}$

This table shows highly statistically significant difference between the patients' and control groups according to EFT at baseline.

There was a high significant difference between both groups regarding EFT, and significant difference regarding total cholesterol and LDLc. While there was no statistical difference between both groups as regard the other variables as illustrated in table (4).

Table (4): Comparison between patients' and control groups regarding the laboratory and clinical data at the baseline.

\begin{tabular}{|c|c|c|c|}
\hline Variable & Patients $(n=50)$ & Control $(n=50)$ & p-value \\
\hline FBS (mg/dl) & $83.50 \pm 8.03$ & $82.10 \pm 7.26$ & $>0.05$ \\
\hline 2h PPBS (mg/dl) & $118.82 \pm 13.70$ & $115.30 \pm 12.16$ & $>0.05$ \\
\hline $\mathrm{Hb} \mathrm{A1c} \%$ & $4.96 \pm 0.33$ & $4.88 \pm 0.24$ & $>0.05$ \\
\hline $\mathrm{TG}(\mathrm{mg} / \mathrm{dl})$ & $123.18 \pm 17.09$ & $120.14 \pm 16.49$ & $>0.05$ \\
\hline T.Ch. (mg/dl) & $163.24 \pm 17.24$ & $154.72 \pm 19.45$ & $<0.05^{*}$ \\
\hline HDLc (mg/dl) & $55.82 \pm 4.37$ & $56.40 \pm 4.35$ & $>0.05$ \\
\hline LDLc (mg/dl) & $82.78 \pm 18.19$ & $74.29 \pm 12.02$ & $<0.05^{*}$ \\
\hline Alb./Creat Ratio (mg/gm creat.) & $19.22 \pm 3.50$ & $18.76 \pm 3.80$ & $>0.05$ \\
\hline Uric Acid (mg/dl) & $4.68 \pm 1.24$ & $4.73 \pm 1.02$ & $>0.05$ \\
\hline ALT (U/L) & $26.50 \pm 4.63$ & $25.16 \pm 4.91$ & $>0.05$ \\
\hline AST (U/L) & $27.16 \pm 7.34$ & $25.06 \pm 7.29$ & $>0.05$ \\
\hline Hs-CRP (mg/dl) & $2.89 \pm 0.83$ & $2.75 \pm 0.58$ & $>0.05$ \\
\hline Systolic BP (mmHg) & $120.50 \pm 5.65$ & $120.40 \pm 5.88$ & $>0.05$ \\
\hline Diastolic BP (mmHg) & $75.90 \pm 3.74$ & $75.40 \pm 3.76$ & $>0.05$ \\
\hline $\mathrm{BMI}\left(\mathrm{Kg} / \mathrm{m}^{2}\right)$ & $21.72 \pm 1.71$ & $21.15 \pm 1.50$ & $>0.05$ \\
\hline $\mathrm{WC}(\mathrm{cm})$ & $87.58 \pm 8.27$ & $86.70 \pm 7.69$ & $>0.05$ \\
\hline EFT (mm) & $5.50 \pm 1.13$ & $3.39 \pm 0.75$ & $k 0.001 * *$ \\
\hline
\end{tabular}

p-value $>0.05 \mathrm{NS} ; *$ p-value $<0.05 \mathrm{~S} ; * *$ p-value $<0.001 \mathrm{HS}$

This table shows statistically significant difference between the patients' and control groups according to T.C., LDLc and EFT. 
Mohammed Abdel-Hassib et al.

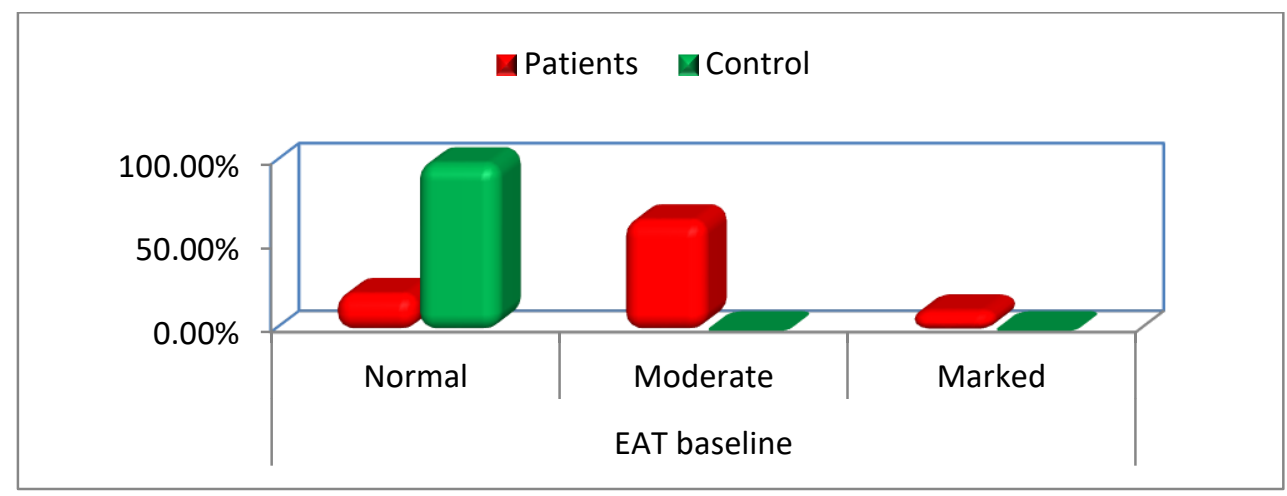

Fig. (1): Bar chart between patients' and control groups according to EFT at baseline.

The same variables were repeated only for the patients' group after 18 months and the results were compared to their baseline results. The U/S grading of HS between the baseline and after 18 months follow up in the patients' group were compared and showed a significant difference as showed in table (2) and figure (3).

Also, there was a significant difference regarding the FBS, HbA1c, TG, uric acid, systolic and diastolic BP, WC and EFT when the baseline results were compared to the follow up results in the patients' group as demonstrated in tables (5 and 6) and illustrated in figure (1 and 2). The remaining variables showed no statistical difference as demonstrated in table (5).

Table (5): Comparison between the laboratory and clinical data in the patients' group at baseline and after 18 months.

\begin{tabular}{|l|c|c|c|c|}
\hline Variable & At baseline & After 18 months & Diff. & p-value \\
\hline FBS (mg/dl) & $83.50 \pm 8.03$ & $98.30 \pm 15.66$ & 14.8 & $<0.001^{* *}$ \\
\hline 2h PPBS (mg/dl) & $118.82 \pm 13.70$ & $124.72 \pm 17.93$ & 5.9 & $>0.05$ \\
\hline Hb A1c \% & $4.96 \pm 0.33$ & $5.33 \pm 0.58$ & 0.37 & $<0.001^{* *}$ \\
\hline TG (mg/dl) & $123.18 \pm 17.09$ & $154.62 \pm 34.15$ & 31.44 & $<0.001^{* *}$ \\
\hline T.Ch. (mg/dl) & $163.24 \pm 17.24$ & $167.52 \pm 23.78$ & 4.28 & $>0.05$ \\
\hline HDLc (mg/dl) & $55.82 \pm 4.37$ & $52.02 \pm 6.86$ & -3.80 & $>0.05$ \\
\hline LDLc (mg/dl) & $82.78 \pm 18.19$ & $84.58 \pm 3.94$ & 1.79 & $>0.05$ \\
\hline Alb./Creat Ratio (mg/gm creat.) & $19.22 \pm 3.50$ & $20.88 \pm 1.98$ & 1.66 & $>0.05$ \\
\hline Uric Acid (mg/dl) & $4.68 \pm 1.24$ & $5.86 \pm 1.42$ & 1.18 & $<0.001^{* *}$ \\
\hline ALT (U/L) & $26.50 \pm 3.63$ & $31.36 \pm 1.26$ & 4.86 & $>0.05$ \\
\hline AST (U/L) & $27.16 \pm 334$ & $30.58 \pm 4.63$ & 3.42 & $>0.05$ \\
\hline Hs-CRP (mg/dl) & $2.89 \pm 0.83$ & $3.61 \pm 0.20$ & 0.72 & $>0.05$ \\
\hline Systolic BP (mmHg) & $120.50 \pm 5.65$ & $123.10 \pm 5.70$ & 2.6 & $<0.001^{* *}$ \\
\hline Diastolic BP $(\mathrm{mmHg})$ & $75.90 \pm 3.74$ & $77.20 \pm 3.80$ & 1.3 & $<0.001^{* *}$ \\
\hline BMI (Kg/m $\left.{ }^{2}\right)$ & $21.72 \pm 1.71$ & $22.14 \pm 1.83$ & 0.42 & $>0.05$ \\
\hline WC (cm) & $87.58 \pm 8.27$ & $96.46 \pm 10.84$ & 8.88 & $<0.001^{* *}$ \\
\hline EFT (mm) & $5.50 \pm 1.13$ & $6.40 \pm 1.30$ & 0.9 & $<0.001^{* *}$ \\
\hline
\end{tabular}

p-value $>0.05 \mathrm{NS} ; * *$ p-value $<0.001 \mathrm{HS}$

Table (6): Comparison between baseline and after 18 months according to EAT in patients' group.

\begin{tabular}{|l|c|c|c|}
\hline EFT & At baseline & After 18 months & \multirow{2}{*}{ p-value } \\
\hline Normal & $11(22.0 \%)$ & $7(14.0 \%)$ & \multirow{2}{*}{$<0.05^{*}$} \\
\cline { 1 - 3 } Moderate & $33(66.0 \%)$ & $22(44.0 \%)$ & \multirow{2}{*}{} \\
\cline { 1 - 3 } Marked & $6(12.0 \%)$ & $21(42.0 \%)$ & \\
\hline
\end{tabular}

$\mathrm{x}^{2}$ : Chi-square test; *p-value $<0.05 \mathrm{~S}$ 


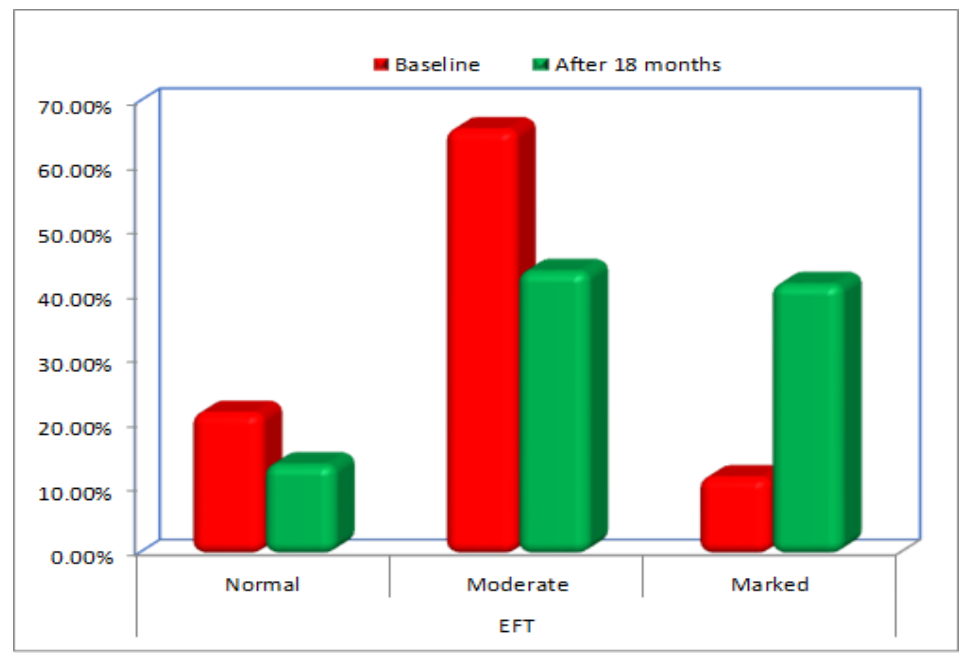

Fig. (2): Bar chart between baseline and after 18 months according to EFT in patients' group.

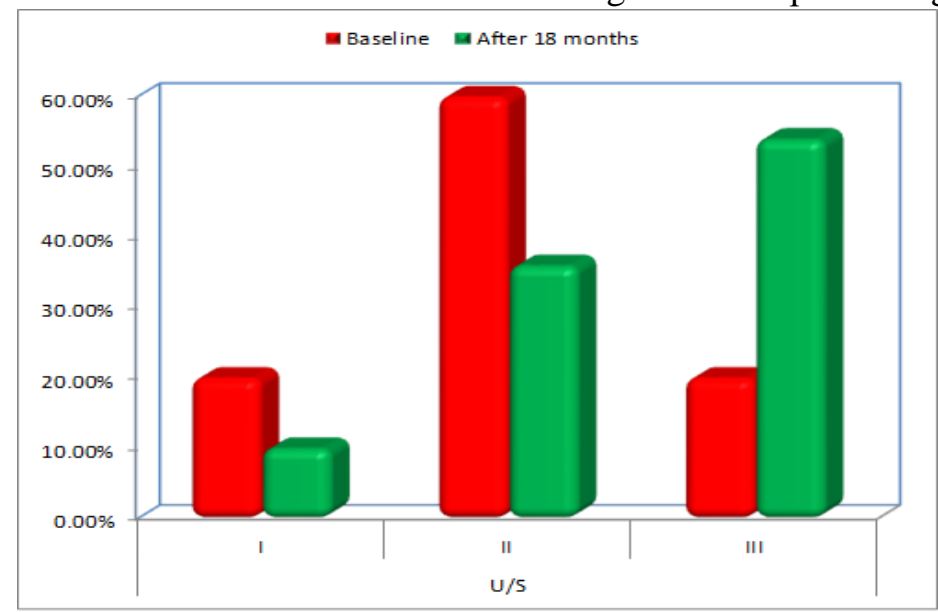

Fig. (3): Bar chart between baseline and after 18 months regarding the degree of HS by U/S in patients' group.

Correlation study between EFT and the other variables at baseline and after 18 months wasdone (As illustrated in table 7) and revealed the following:

- Positive and significant correlation between EFT at baseline with FBS, 2h PPBS, HbA1c, TG, T.Ch., LDLc, HDLc, Alb./Creat Ratio, ALT, AST, BMIand WC.

- No correlation with uric acid hsCRP and systolic and diastolic BP.

- Positive and significant correlation between EFT after 18 months with FBS, 2h PPBS, Hb A1c, TG, T.Ch., LDLc, HDLc, Alb./Creat Ratio, ALT, AST, Hs-CRP, diastolic BP, BMI and WC.

- No correlation with uric acid and systolic BP.

A similar correlation study between U/S degree of HS and the other variables at baseline and after
18 months was done (As illustrated in table 8 )and revealed the following:

- Positive and significant correlation between U/S at baseline with FBS, $2 \mathrm{~h}$ PPBS, Hb A1c, TG, T.Ch., LDLc, Alb./Creat Ratio, ALT, AST, systolic BP, BMI and WC.

- No correlation with uric acid hsCRP and diastolic BP.

- Positive and significant correlation between U/S after 18 months with FBS, 2h PPBS, Hb A1c, TG, T.Ch., LDLc, Alb./Creat Ratio, ALT, Diastolic $\mathrm{BP}, \mathrm{BMI}$ and WC.

- No correlation with uric acid, AST, hsCRP and systolic BP.

The correlation between EFT and U/S degree of HS both at baseline and after 18 months showed positive and significant correlation as illustrated in table (9) and in the scatter plot figures

(4) and (5). 
Table (2): Correlation between EFT with all parameters, using Pearson Correlation Coefficient in patients group.

\begin{tabular}{|c|c|c|c|c|}
\hline \multirow{3}{*}{ Parameters } & \multicolumn{4}{|c|}{ EFT } \\
\hline & \multicolumn{2}{|c|}{ At baseline } & \multicolumn{2}{|c|}{ After 18 months } \\
\hline & $\mathbf{r}$ & p-value & $\mathbf{r}$ & p-value \\
\hline Age (years) & -0.044 & $>0.05$ & -0.084 & $>0.05$ \\
\hline FBS (mg/dl) & 0.539 & $<0.001 * *$ & 0.457 & $<0.001 * *$ \\
\hline 2h PPBS (mg/dl) & 0.484 & $<0.001 * *$ & 0.406 & $<0.001 * *$ \\
\hline $\mathrm{Hb}$ A1c $\%$ & 0.506 & $<0.001 * *$ & 0.504 & $<0.001 * *$ \\
\hline TG (mg/dl) & 0.500 & $<0.001 * *$ & 0.602 & $<0.001 * *$ \\
\hline T.Ch. (mg/dl) & 0.504 & $<0.001 * *$ & 0.424 & $<0.001 * *$ \\
\hline HDLc (mg/dl) & -0.515 & $<0.001 * *$ & -0.321 & $0.013^{*}$ \\
\hline LDLc (mg/dl) & 0.507 & $<0.001 * *$ & 0.382 & $0.007 *$ \\
\hline Alb./Creat Ratio (mg/gm creat.) & 0.425 & $0.002 *$ & 0.444 & $<0.001 * *$ \\
\hline Uric Acid (mg/dl) & 0.144 & $>0.05$ & 0.213 & $>0.05$ \\
\hline ALT (U/L) & 0.445 & $<0.001 * *$ & 0.385 & $<0.001 * *$ \\
\hline AST (U/L) & 0.368 & $0.009 *$ & 0.287 & $0.024^{*}$ \\
\hline Hs-CRP (mg/dl) & -0.066 & $>0.05$ & 0.317 & $0.038^{*}$ \\
\hline Systolic BP mmHg & 0.268 & $>0.05$ & 0.236 & $>0.05$ \\
\hline Diastolic BP mmHg & 0.083 & $>0.05$ & 0.332 & $0.018^{*}$ \\
\hline $\mathrm{BMI} \mathrm{Kg} / \mathrm{m}^{2}$ & 0.445 & $<0.001 * *$ & 0.395 & $0.004 * *$ \\
\hline $\mathrm{WC}(\mathrm{cm})$ & 0.769 & $<0.001 * *$ & 0.856 & $<0.001 * *$ \\
\hline
\end{tabular}

r-Pearson Correlation Coefficient

p-value $>0.05 \mathrm{NS}$; ${ }^{*}$-value $<0.05 \mathrm{~S}$; $* *$ p-value $<0.001 \mathrm{HS}$

Table (3): Correlation between U/S degrees of HS with all parameters, using Pearson Correlation Coefficient in patients' group.

\begin{tabular}{|c|c|c|c|c|}
\hline \multirow{3}{*}{ Parameters } & \multicolumn{4}{|c|}{ U/S degrees of $\mathrm{HS}$} \\
\hline & \multicolumn{2}{|c|}{ At baseline } & \multicolumn{2}{|c|}{ After 18 months } \\
\hline & $\mathbf{r}$ & p-value & $\mathbf{r}$ & p-value \\
\hline Age (years) & -0.009 & $>0.05$ & -0.102 & $>0.05$ \\
\hline FBS $(\mathrm{mg} / \mathrm{dl})$ & 0.486 & $<0.001 * *$ & 0.435 & $0.002 *$ \\
\hline 2h PPBS (mg/dl) & 0.357 & $<0.05 *$ & 0.374 & $0.008^{*}$ \\
\hline $\mathrm{Hb} \mathrm{A1c} \%$ & 0.419 & $<0.05^{*}$ & 0.448 & $<0.001 * *$ \\
\hline TG $(\mathrm{mg} / \mathrm{dl})$ & 0.436 & $<0.05^{*}$ & 0.541 & $<0.001 * *$ \\
\hline T.Ch. (mg/dl) & 0.398 & $<0.05^{*}$ & 0.366 & $0.009 *$ \\
\hline HDLc (mg/dl) & -0.351 & $<0.05^{*}$ & -0.260 & $0.041^{*}$ \\
\hline LDLc (mg/dl) & 0.380 & $<0.05^{*}$ & 0.322 & $0.014 *$ \\
\hline Alb./Creat Ratio (mg/gm creat.) & 0.305 & $<0.05^{*}$ & 0.354 & $0.012^{*}$ \\
\hline Uric Acid $(\mathrm{mg} / \mathrm{dl})$ & 0.080 & $>0.05$ & 0.159 & $>0.05$ \\
\hline ALT (U/L) & 0.427 & $<0.05^{*}$ & 0.298 & $0.024 *$ \\
\hline AST (U/L) & 0.361 & $<0.05^{*}$ & 0.213 & $>0.05$ \\
\hline $\mathrm{Hs}-\mathrm{CRP}(\mathrm{mg} / \mathrm{dl})$ & -0.077 & $>0.05$ & 0.212 & $>0.05$ \\
\hline Systolic BP mmHg & 0.339 & $<0.05^{*}$ & 0.248 & $>0.05$ \\
\hline Diastolic BP mmHg & 0.128 & $>0.05$ & 0.331 & $0.019 *$ \\
\hline $\mathrm{BMI} \mathrm{Kg} / \mathrm{m}^{2}$ & 0.370 & $<0.05^{*}$ & 0.304 & $0.019^{*}$ \\
\hline $\mathrm{WC}(\mathrm{cm})$ & 0.680 & $<0.001 * *$ & 0.745 & $<0.001 * *$ \\
\hline
\end{tabular}

r-Pearson Correlation Coefficient, p-value $>0.05 \mathrm{NS} ;{ }^{*}$ p-value $<0.05 \mathrm{~S} ;{ }^{* *}$-value $<0.001 \mathrm{HS}$ 
Hepatic and Epicardial Fat as Early Strong Predictors for Metabolic Syndrome

Table (4): Correlation between U/S degrees of HS with EFT, using Pearson correlation coefficient in patients' group.

\begin{tabular}{|l|c|c|c|}
\hline \multicolumn{2}{|c|}{} & U/S- baseline & U/S- after 18 month \\
\hline \multirow{2}{*}{ EFT at baseline } & $\mathrm{r}$ & 0.918 & 0.798 \\
\cline { 2 - 4 } & $\mathrm{p}$-value & $<0.001^{* *}$ & $<0.001^{* *}$ \\
\hline \multirow{2}{*}{ EFT after 18 months } & $\mathrm{r}$ & 0.838 & 0.930 \\
\cline { 2 - 4 } & $\mathrm{p}$-value & $<0.001^{* *}$ & $<0.001^{* *}$ \\
\hline
\end{tabular}

r-Pearson Correlation Coefficient

**p-value $<0.001 \mathrm{HS}$

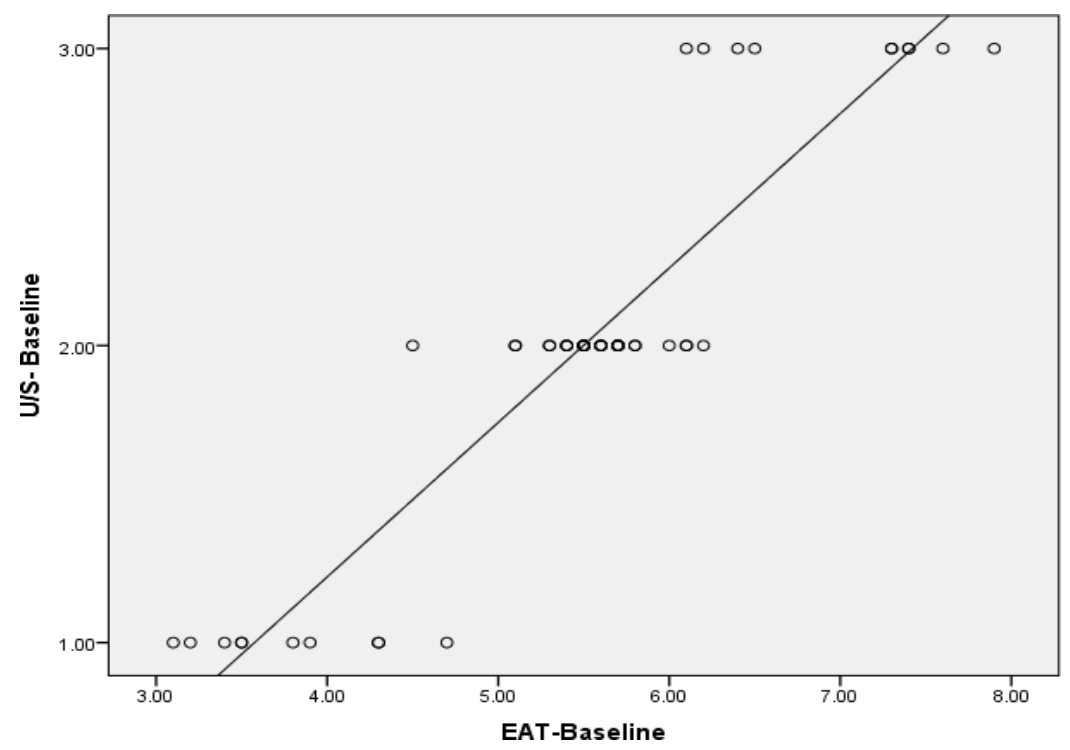

Fig. (4): Scatter plot between EFT at baseline and U/S degree of HS at baseline.

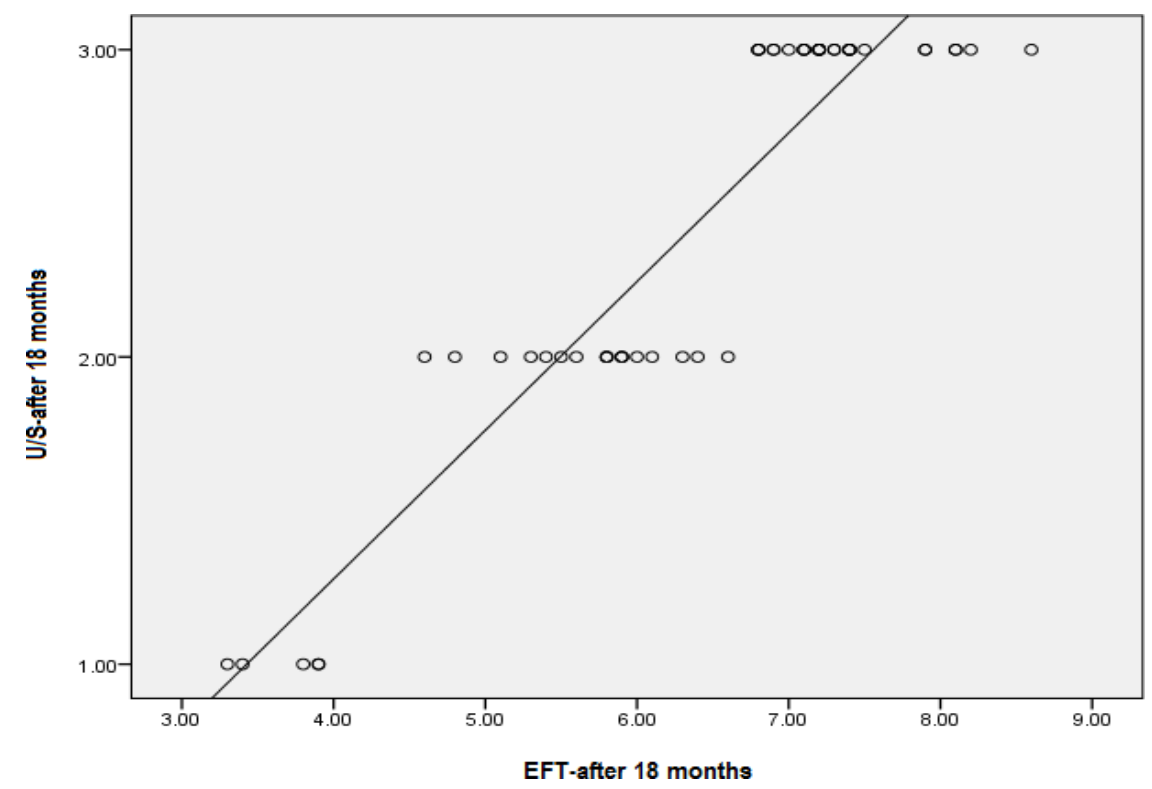

Fig. (5): Scatter plot between EFT and U/S degrees of HS after 18 months. 


\section{DISCUSSION}

Our study revealed a high significant difference between EFT in the patients' group and the control group. Moreover, there was a high significant positive correlation between EFT and degrees of HS in the patients' group both at baseline and after 18 months follow up that strongly supports the co-existence of both variables.

Also, there was a significant positive correlation between EFT and some components of MetS that developed after follow-up of the patients' group.

Many studies done to determine the association of EAT and HS with the components of MetS. In an earlier study by Iacobellis ${ }^{[15]}$ who reported that, the EAT thickness of 9.5 and $7.5 \mathrm{~mm}$ maximize the sensitivity and specificity to predict MetS in men and women respectively. Median values of 9.5 and $7.5 \mathrm{~mm}$ should be considered the threshold values for high-risk echocardiographic EAT thickness in white men and women while the control group showed $4.0 \pm 0.1 \mathrm{~mm}$.

In concordance with our results, multiple studies conducted by Kaya et al., ${ }^{[16]}$, Torres et al. ${ }^{[7]}$ and Wang et al. [17] concluded that EAT values (measured either echocardiographically or by CT) were linked to anthropometric and clinical metabolic syndrome and can be used for predicting components of MetS.

Of the same view, in a meta-analysis by Rabkin ${ }^{[8]}$, EAT was $7.5 \pm 0.1 \mathrm{~mm}$ in thickness in the MetS patients compared to $4.0 \pm 0.1 \mathrm{~mm}$ in controls, and in another meta-analysis conducted by Pierdomenico et al. ${ }^{[6]}$, using 2027 subjects (1030 of them with MetS) revealed that EAT mass of these patients was significantly thicker than that of controls suggesting the strong association between the echocardiographic evaluation of this tissue and the clinical and anthropometric components ofMetS.

Similarly; Malavazos et al. ${ }^{[5]}$ reported that EAT thickness was significantly higher $(9.760$ vs. 8.607 vs. $7.561 \mathrm{~mm}, \mathrm{P}<0.01$ ) in subjects with (severe NAFLD) than those with (moderateNAFLD) and no liver steatosis (no NAFLD) respectively.

Our results also correspond to those of Marcos et al. ${ }^{[18]}$ which showed some EAT thickness values to have a greater significance in the MetS group than in the control group $(5.69 \pm 1.12$ vs. 3.52 $\pm 0.80 \mathrm{~mm}, \mathrm{p}=0.0001)$. When comparing the biochemical variables between participants; the MetS study group revealed significantly higher plasma levels of FBS $(\mathrm{p}=0.005)$, basal insulin ( $\mathrm{p}=$ $0.0001)$, non-HDL-c $(\mathrm{p}=0.002)$, triglycerides $(\mathrm{p}=$ $0.0001), \mathrm{TG} / \mathrm{HDL}-\mathrm{c}$ ratio $(\mathrm{p}=0.0001)$, and hs-CRP ( $\mathrm{p}=0.0001)$, as well as lower plasma levels of HDL$\mathrm{c}(\mathrm{p}=0.001)$ than the control group.

In agreement with our results, Iacobellis et al. ${ }^{[19]}$ entered EAT thickness, WC and BMI, in a multiple regression analysis to predict ultrasound measured liver steatosis; the dependent variable. EAT thickness was the best independent correlate of liver steatosis. Moreover, when patients were stratified by the ultrasound score those with severe fatty liver infiltration presented with the highest amount of cardiac fat accumulation. Because it can be difficult to measure abdominal adipose tissue, it may be more efficient to determine these risk factorsby measuring EFT. So, they suggested that epicardial fat is a good predictor of liver steatosis in obese subjects.

Kyoung et al. ${ }^{[20]}$ also suggested that echocardiographic EAT is a better predictor of ultrasound-measured NAFLD in MetS than BMI or WC and is associated with MetS. Hence, it can be a predictor of other cardiometabolic diseases.

The association between EAT, metabolic and clinical variables, ultrasonographic steatosis grades and FLI are reported by Fracanzani et al. ${ }^{[21]}$ and found that age, male gender, BMI, diastolic B.P., the degree of ultrasonographic steatosis, FLI were independently associated with EAT. They concluded that, in patients with HS, EAT is associated with the severity of liver and vasculardamage besides with the known metabolic risk factors.

In addition, a previous study by Katsiki etal. ${ }^{[22]}$ have shown that EAT is increased inhypertensive individuals, PCO, type 2 diabetesmellitus (DM), and even type 1 (DM) associated with central obesity.

Our study results also revealed a positiveand significant correlation between EFT and FBS, $2 \mathrm{~h}$ PPBS, HbA1c, TG, T.Ch., LDLc, HDLc,

Alb./Creat Ratio, ALT, diastolic BP, BMI and WC, while no correlation with uric acid, AST, hsCRP and systolic BP was found.

The above described results are also coincide with the results of Dilek et al. ${ }^{[23]}$ and Fernández et al., ${ }^{[24]}$ that concluded that; Highly significant correlation between degree of EATthickness with both systolic and diastolic BP and thelevels of FBS, BMI, WC, HS, insulin level, LDLc, 
TG, and transaminases, but no correlation with HDLc.

In quite agreement with our results, Marcos et al. ${ }^{[18]}$ revealed that EAT showed a statistically significant positive correlation with BMI $(\mathrm{r}=0.661$; $\mathrm{p}=0.0001) ; \mathrm{WC}(\mathrm{r}=0.664 ; \mathrm{p}=0.0001)$, systolic BP $(\mathrm{r}=0.607 ; \mathrm{p}=0.0001)$, and diastolic $\mathrm{BP}(\mathrm{r}=0.447$; $\mathrm{p}=0.0001)$. Also, It showed a statistically significant positive correlation with insulin plasma levels $(\mathrm{r}=$ $0.505 ; \mathrm{p}=0.0001), \mathrm{TG} / \mathrm{HDL}-\mathrm{c}$ ratio $(\mathrm{r}=0.447 ; \mathrm{p}=$ $0.0001)$, and non-HDL-c ( $\mathrm{r}=0.353 ; \mathrm{p}=$

$0.07)$; and a statistically significant negative correlation with plasma concentrations of adiponectin $(\mathrm{r}=0.499 ; \mathrm{p}=0.0001)$. There was no correlation between hs-CRP and EAT thickness.

Of the same view, Lai et al. ${ }^{[25]}$ reported a positive correlation between EAT and fasting glucose, insulin resistance, and alanine transaminase levels. Wang et al., ${ }^{[26]}$ also, found a strongcorrelation between fasting plasma glucose and EATmeasured with CT or echocardiography.

Kankaanpää et al. ${ }^{[27]}$ showed that, EAT (measured with MRI), and the degree of HS were correlated with abdominal adiposity and hypertriglyceridemia.

Although hyperuricemia has been suggested as an independent risk factor for NAFLD, Sirota et al. [28] did not explore any relationship between epicardial fat and uric acid in subjects with NAFLD.

Similar to our results, Dicker et al. ${ }^{[29]}$ conducted a study in non-obese subjects, and reported that EAT thickness $\geq 2.4 \mathrm{~mm}$ is associated with an increased risk of high BP (odds ratio 1.39; 95\% CI: 1.033-1.992).

This result coincides with that of the studyby Hüseyin et al., ${ }^{[30]}$ who concluded that, EFT over $>5.5 \mathrm{~mm}$ predicts newly diagnosis hypertension with $63 \%$ sensitivity and $84 \%$ specificity; and with that of Eroglu et al. ${ }^{[31]}$ who previously reported a cutoff value of EAT over the $0.7 \mathrm{~cm}$ related the severity of hypertension.

Also our results corresponds to that of a meta-analysis by Rabkin ${ }^{[8]}$, evaluating the relationship between epicardial fat, indices of obesity and MetS, demonstrated that, EAT thickness significantly correlated independently with each of the components of MetS (Systolic BP, HDL-c, fasting glucose), however, unlikely to our results, the magnitude of this relationship was considerably and significantly lower than that between epicardial fat and BMI.
On the contrary to our results, Aydin et al. ${ }^{[14]}$ evinced no independent relationship between EAT thickness and TG/HDL-c ratio.

\section{CONCLUSION}

1- There is strong correlation between HS and EFT. HS is a good predictor for EFT.

2- HS and EFT are early and strong predictor of MetS and cardiovascular risk.

3- BMI and WC alone aren't early and sensitive enough, as the HS and EFT, for prediction of MetS.

\section{RECOMMENDATIONS}

We believe that the combined assessment of HS and EFT, have the potential for immediate clinical application as they are easy and applicable indaily practice.

\section{REFERENCES}

1. Cheung O, Kapoor A, Puri P, Sistrun S, Luketic VA, Sargeant $\mathbf{C C}(\mathbf{2 0 0 7 )}$ : The impact of fat distribution on the severity of nonalcoholic fatty liver disease and metabolic syndrome. Hepatology, 46(4):1091-1100.

2. Iacobellis G, Bianco AC (2011): Epicardial adipose tissue: emerging physiological, pathophysiological and clinical features. Trends Endocrinol Metab., 22:450- 457.

3. Nagy E, Jermendy AL, Merkely B, Maurovich HP(2017): Clinical importance of epicardial adipose tissue. Arch Med Sci. ,13(4):864-874.

4. Smits MM, Ioannou GN, Boyko EJ (2013): Nonalcoholic fatty liver disease as an independent manifestation of the metabolic syndrome: results of a US national survey in three ethnic groups. J Gastroenterol Hepatol .,28:664-670.

5. Malavazos AE, Di Leo G, Secchi F (2010):Relation of echocardiographic epicardial fat thickness and myocardial fat. Am J Cardiol ., 105:1831-1835.

6. Pierdomenico SD, Pierdomenico AM, Cuccurullo F, Iacobellis G (2013): Metaanalysis of the relation of echocardiographic epicardial adipose tissue thickness and the metabolic syndrome. Am J Cardiol.,111(1):73-8.

7. Torres C, Lima-Martínez MM, Rosa FJ, Guerra E, Paoli M, Iacobellis G (2011): Epicardial adipose tissue and its association to plasma adrenomedullin levels in patients with metabolic syndrome. Endocrinol Nutr.,58(8):401-8.

8. Rabkin SW (2014): The relationship between epicardial fat and indices of obesity and the metabolic syndrome: a systematic review and meta- analysis. Metab Syndr Relat Disord.,12(1):31-42. 
9. Sacks HS, Fain JN (2007): Human epicardial adipose tissue: a review. Am Heart J., 153:907-917.

10. Verhagen SN, Visseren FL (2011): Perivascular adipose tissue as a cause of atherosclerosis. Atherosclerosis ,214(1):3-10.

11. Gastaldelli A, Basta $G$ (2010): Ectopic fat and cardiovascular disease: what is the link? Nutr Metab Cardiovasc Dis.,20:481-490.

12. Ouchi N, Kihara S, Funahashi T, Nakamura T, Nishida M, Kumada M (2003): Reciprocal association of C-reactive protein with adiponectin in blood stream and adipose tissue. Circulation, 107(5):671-4.

13. Liang KW, Tsai IC, Lee WJ, Lee IT, Lee WL, Lin SY, Wan CJ, Fu CP, Ting CT, Sheu WH (2011): MRI measured epicardial adipose tissue thickness at the right AV groove differentiates inflammatory status in obese men with metabolic syndrome. Obesity ,20(3):525-532.

14. Aydin H, Toprak A, Deyneli O, Yacizi D, Tarçin O, Sancak S (2010): Epicardial fat tissue thickness correlates with endothelial dysfunction and other cardiovascular risk factors in patients with metabolic syndrome. Metab Syndr Relat Disord., 8(3):229-34.

15. Iacobellis G1, Willens HJ, Barbaro G, Sharma AM(2008): Threshold values of high-risk echocardiographic epicardial fat thickness. Obesity ,16(4):887-92.

16. Kaya B, Kaya BC, Karakas EY (2015): Usefulness of the epicardial fat tissue thickness as a diagnostic criterion for geriatric patients with metabolic syndrome. J Geriatr Cardiol .,12:373-377.

17. Wang TD, Lee WJ, Shih FY, Huang CH, Chen WJ, Lee YT, Shih TT, Chen MF (2010): Association of epicardial adipose tissue with coronary atherosclerosis is region-specific and independent of conventional risk factors and intra- abdominal adiposity. Atherosclerosis, 213(1):279- 287.

18. Marcos M. Gabriel LM and Rodolfo JH (2014): Donis Mariela Paoli. Epicardial adipose tissue thickness and its association with adiponectin in metabolic syndrome patients from Mérida, Venezuela Endocrinol Metab., 58:4.

19. Iacobellis G, Giorgio BL and Giuseppe B (2014): Epicardial fat thickness and nonalcoholic fatty liver disease in obese subjects. Obesity ,22:332-336.

20. Kyoung Im Cho, Eun AJ, Sang HC and Bo HK (2017): Metabolic syndrome and related disorders. Mary Ann Liebert, Inc.

21. Fracanzani AL, Pisano G, Consonni D, Tiraboschi S, Baragetti A, and Bertelli C (2016): Epicardial adipose tissue (EAT) thickness is associated with cardiovascular and liver damage in nonalcoholic fatty liver disease, 1(9):e0162473.

22. Katsiki N, Mikhailidis DP, Wierzbicki AS (2013): Epicardial fat and vascular risk: A narrative review. Curr Opin Cardiol.,28:458-463.

23. Dilek A, Aysel GT, Sabiye $Y$ and Tamer NK (2015): The relationship between epicardial fat tissue thickness and visceral adipose tissue in lean patients with polycystic ovary syndrome. J Ovarian Res., 8:71.

24. Fernández MJ, Basurto AL, Córdova PN, Vázquez AL, Tepach G N and Vega GS (2014): Epicardial adipose tissue is associated with visceral fat, metabolic syndrome, and insulin resistance in menopausal women. Rev. Esp Cardiol., 67(6):436441.

25. Lai YH, Yun CH, Yang FS (2012): Epicardial adipose tissue relating to anthropometrics, metabolic derangements and fatty liver disease independently contributes to serum high-sensitivity C-reactive protein beyond body fat composition: a study validated with computed tomography disease. J. Am. Soc. Echocardiogr.,25:234-241.

26. Wang TD, Lee WJ and Shih FY (2009): Relations of epicardial adipose tissue measured by multidetector computed tomography to components of the metabolic syndrome are region-specific and independent of anthropometric indexes and intraabdominal visceral fat. J Clin Endocrinol Metab., 94: 662-9.

27. Kankaanpää M, Lehto HR, Pärkkä JP (2006): Myocardial triglyceride content and epicardial fat mass in human obesity: relationship to leftventricular function and serum free fatty acid levels. J Clin Endocrinol Metab .,91:4689-4695.

28. Sirota JC, McFann K, Targher G (2013):Elevated serum uric acid levels are associated with nonalcoholic fatty liver disease independently of metabolic syndrome features in the United States:liver ultrasound data from the National Health and Nutrition Examination Survey. Metabolism,62:392399.

29. Dicker D, Atar E, Kornowski R, Bachar GN (2013): Increased epicardial adipose tissue thickness as a predictor for hypertension: a cross-sectional observational study. J Clin Hypertens .,15(12):893-8.

30. Hüseyin ÖH, Katlandur KO, Ahmet KA and Osman KS (2017): epicardial fat thickness is an important diagnostic marker for newly diagnosed essential hypertension. Research Article - Biomedical Research ,28: 1.

31. Eroglu S, Sade LE, Yildirir A, Demir $O$ and Muderrisoglu H (2013): Association of epicardial adipose tissue thickness by echocardiography and hypertension. Turk Kardiyol Dern Ars., 41:115-122. 